\title{
Tackling bovine TB
}

1. Mark Jones,

2. Fiona Dalzell,

3. Richard Edwards,

4. Phill Elliott,

5. Geoff Hale,

6. Sophie Hill,

7. Jo Hinde,

8. Andrew Knight,

9. Joanne Lewis,

10. Andre Menache,

$11 . \quad J u d y$ Puddifoot,

12. Richard Saunders,

13. Guda van der Burgt,

14. Iain McGill,

15. Elisa Allen,

16. Dominic Dyer,

17. Philip Mansbridge,

18. Lorraine Platt and

19. Toni Shephard

On 18 December Defra revealed that during 2018, 32,601 badgers were killed, bringing the total number slaughtered under licence since 2013 to almost $67,000 .^{1}$

'Effectiveness' claims relate not to the impact on cattle TB, but rather to the ability of the contracted shooters to kill sufficient badgers to satisfy their licence requirements, which they can hardly fail to reach given that target numbers are 'adjusted' by Natural England part-way through the culls according to the contractors' progress.

Sixty per cent of the badgers have been killed by 'controlled shooting', a method rejected by both the government's Independent Expert Panel ${ }^{2}$ and the BVA $^{3}$ because of animal welfare concerns. During 2018 Natural England directly monitored just 89 ( 0.43 per cent) of controlled shooting events.

It is deplorable that the chief veterinary officer (CVO) continues to support the roll-out of a policy that permits controlled shooting, when veterinary organisations have condemned the method on animal welfare grounds. It is particularly concerning that the CVO appears to have deflected responsibility for humaneness to Natural England's chief scientist who, as far as we are aware, has no background in animal welfare science.

It is also unacceptable for government to attribute reductions in herd bovine TB (bTB) incidents over the first four years of culling in the original 'pilot' cull zones to its badger culling policy. ${ }^{4}$ Independent analysis of this and more recent data from the Gloucestershire pilot cull zone ${ }^{5}$ indicate that new herd 
incidence is rising sharply, with 22 herd breakdowns in the 12 months to September 2017 (an increase of 29.4 per cent when compared to the 17 breakdowns reported by APHA for the previous 12 months). Analysis of the 2018 figures indicates that both incidence and prevalence are now rising even faster, with a further 24 herd breakdowns occurring between 1 January and 5 December 2018.

To date, the government and its officials cite data that are two years out of date, but have declined to comment on this emerging evidence that, far from resulting in a substantial disease control benefit, badger culls may be leading to a sharp increase in bTB in cattle.

Natural England's chief scientist and the UK's CVO continue to endorse a failing and inhumane policy, bringing their offices into serious disrepute. We urge them, and the BVA, to reconsider their support for further badger culling, and instead focus on the actual cause of bTB's epidemic spread - a cattle skin test with a sensitivity of only 50 per cent, ${ }^{6,7}$ and the ongoing problems associated with cattle movements and on-farm biosecurity.

\section{References}

1. Defra. Bovine TB: summary of badger control monitoring during 2018. 2018. www.gov.uk/government/publications/bovine-tb-summary-ofbadger-control-monitoring-during-2018 (accessed 22 January 2019)

2. Defra. Pilot badger culls in Somerset and Gloucestershire: report by the Independent Expert

Panel. 2014. www.gov.uk/government/publications/pilot-badger-culls-insomerset-and-gloucestershire-report-by-the-independent-expertpanel (accessed 22 January 2019)

3. Anon. Badger culling: BVA withdraws support for controlled shooting. Vet $\operatorname{Rec} 2015 ; 176: 423$

4. Defra. Effectiveness of badger culls. Vet Rec 2018;183:538

5. APHA/ERGO. Mapping bovine TB (bTB) in England and Wales. 2019. www.ibtb.co.uk (accessed 22 January 2019)

6. Nuñez-Garcia J,

1. Downs $\mathrm{SH}$,

2. Parry JE, et al

. Meta-analyses of the sensitivity and specificity of ante-mortem and postmortem diagnostic tests for bovine tuberculosis in the UK and Ireland. Prev Vet Med 2018;153:94-107

Lahuerta-Marin A,

3. Milne MG,

4. McNair J, et al 
. Bayesian latent class estimation of sensitivity and specificity parameters of diagnostic tests for bovine tuberculosis in chronically infected herds in Northern Ireland. Vet J 2018;238:15-21 